\title{
Status of pulmonary fungal infections among individuals with clinical signs of pulmonary tuberculosis at a University Teaching Hospital in Southwestern Uganda.
}

\section{Israel Kiiza Njovu ( $\square$ israelkin25@gmail.com )}

Department of Microbiology, Faculty of Medicine, Mbarara University of Science and Technology, Mbarara, Uganda

\section{Benson Musinguzi}

Department of Medical Laboratory Science, Faculty of Health Sciences, Muni University, Arua, Uganda https://orcid.org/0000-0002-1211-4617

\section{James Mwesigye}

Department of Microbiology, Faculty of Medicine, Mbarara University of Science and Technology, Mbarara, Uganda

\section{Kennedy Kassaza}

Department of Microbiology, Faculty of Medicine, Mbarara University of Science and Technology, Mbarara, Uganda

\section{Joseph Turigurwa}

Department of Microbiology, Faculty of Medicine, Mbarara University of Science and Technology, Mbarara, Uganda

\section{Edwin Nuwagira}

Department of Internal Medicine, Faculty of Medicine, Mbarara University of Science and Technology, Mbarara, Uganda Joel Bazira

Department of Microbiology, Faculty of Medicine, Mbarara University of Science and Technology, Mbarara, Uganda

\section{Taseera Kabanda}

Department of Microbiology, Faculty of Medicine, Mbarara University of Science and Technology, Mbarara, Uganda

\section{Moses Mpeirwe}

Department of Microbiology, Faculty of Medicine, Mbarara University of Science and Technology, Mbarara, Uganda

\section{Lucas Ampaire}

Department of Medical Laboratory Sciences, Faculty of medicine, Mbarara University of Science and Technology, Mbarara, Uganda 


\section{Andrew Mutekanga}

Department of Internal Medicine, Faculty of Medicine, Mbarara University of Science and Technology, Mbarara, Uganda

James Kiguli

Department of Microbiology and Immunology, School of Health Sciences, Soroti University, Soroti, Uganda

\section{Beatrice Achan}

Department of Microbiology, School of Biomedical Sciences, Makerere University, Uganda

\section{Herbert Itabangi}

Department of Microbiology and Immunology, Faculty of Health Sciences, Busitema University, Mbale, Uganda

\section{Research Article}

Keywords: fungal infections, pulmonary tuberculosis

Posted Date: May 13th, 2021

DOl: https://doi.org/10.21203/rs.3.rs-523551/v1

License: (c) (i) This work is licensed under a Creative Commons Attribution 4.0 International License. Read Full License 


\section{Abstract}

Background: Pulmonary mycoses are very important diseases of the respiratory tract and are responsible for significant morbidity and mortality rates worldwide. However less attention has been paid to them. In this study we determined the prevalence of pulmonary mycoses and their aetiological agents among individuals with clinical signs of pulmonary tuberculosis at Mbarara Regional Referral Hospital (MRRH).

Method: This was a Laboratory based cross sectional survey in which 113 participants were recruited. Sputum samples were corrected from each study participant. To each sample the following tests were done; Sabouraud Dextrose Agar (SDA) Culture, GeneXpert and Potassium hydroxide ( $\mathrm{KOH})$. Fungal growth of filamentous fungi and yeasts were further examined with LPCB and Germ tube respectively. Generated data was analysed using R studio.

Results: Out of 113 participants, $80(70.8 \%)$ had pulmonary mycoses whilst those with pulmonary tuberculosis were 5 (4.4\%). C. albicans $22.58 \%$ and Aspergillus species $17.20 \%$ ) were the most aetiological agents for pulmonary mycoses identified among others. Of those who were TB GeneXpert positive, $2(1.77 \%)$ of them were co-infected with pulmonary mycoses. We established a prevalence of 57 (71.3\%) for PFI, $3(60.0 \%)$ for MTB in HIV sero positive patients and 18 (22.5\%) for PFI, $0(0.00 \%)$ for MTB in HIV sero negative patients. On the other hand, $2(100 \%)$ sero positive patients were co-infected with both PFI and MTB.

Conclusion: Our findings highlight the medical importance of pulmonary fungal infections among patients suspected for TB. Importantly, the aetiological diversity established here is rich enough to suggest precise examination using different methods. Established scope of aetiological agents is predominated by opportunistic fungi which when superimposed on to certain underlying conditions such as TB, their effects can be fatal. Therefore this possibility presents a need to employ appropriate strategies for prevention, diagnosis, and management in TB suspects.

\section{Introduction}

Pulmonary tuberculosis (PTB) remains a fundamental cause of sub-acute and chronic pulmonary disease and thus a major cause of mortality attributed mainly to Mycobacterium tuberculosis (MTB) complex. MTB claims over 1 million people with about $95 \%$ of these reported in Asia and Africa respectively where a developing country like Uganda lies $(1,2)$. In Africa and Asia, the annual incidence of TB is very high reaching hundreds of cases per 100,000 people (3). In Uganda alone, TB is estimated at a prevalence of 200 per 100,000 people (4); in Mbarara, the prevalence of TB is estimated at 98 per 100,000 people (5). Certainly, MTB is still a major co-morbidity and a leading cause of death among individuals infected with Human Immunodeficiency virus (HIV/AIDS). Additionally, the emergency of multidrug resistant (MDR) and extensively drug resistant (XDR) TB has seriously complicated TB diagnosis, treatment and management. However, amidst all this, TB is not the only pulmonary infection of importance especially with the expansion of at-risk populations worldwide. For instance, the role of 
pulmonary fungal infections has been highlighted recently and the similarity in clinical and radiological characteristics with TB further complicates diagnosis and management of such pulmonary infections (6). Indeed, pulmonary mycoses can mimic and easily be misdiagnosed as TB and vice versa. In this context, fungi have over time gained attention for their recent emerging medical importance worldwide (79). Over the past 30 years now, fungi have transitioned into key aetiological agents for difficult to manage infections, killing at least 1 million people annually; and yet again remain among the most neglected diseases globally. The very serious invasive fungal infections occur in the immune-compromised such as HIV/AIDS, cancer, body transplants among others, in many of whom they complicate and worsen the disease (10). For instance, in Africa, pulmonary fungal infections are reported at about $15-35 \%$ mainly in HIV/TB co infected cohorts. Additionally, the similarity in clinical and diagnostic manifestations between TB and pulmonary mycoses calls for equal attention if management of both infections is to be streamlined (11). This is posing enormous challenges to health care professionals especially in resource limited settings where diagnosis is not precise. It is possible that in numerous cases, missed fungal pulmonary mycoses due to lack of specific clinical manifestations causes a high rate of morbidity and mortality in patients initially suspected and treated for TB. The question at hand here is whether fungal pulmonary infection can be primary or secondary in TB infection. Although this is a challenging question to answer at the moment, through this study we sought to determine the prevalence of pulmonary mycoses, aetiology and PTB, HIV sero positive and fungal co-infections among individuals with clinical signs of pulmonary tuberculosis at TB referral clinical in south western Uganda.

\section{Materials And Methods}

\section{Patients and Samples}

This was a laboratory based cross sectional study that recruited 113 patients clinically suspected for PTB. From these patients, an on-spot early morning sputum sample was collected aseptically in dry widenecked, leak-proof containers from each of the suspected patients referred the TB clinic of Mbarara Regional Referral Hospital (MRRH) between 2019-2020. Sputum samples were then transferred to the Mycology Laboratory Unit at Microbiology Department, Faculty of Medicine, MUST. Other information collected from the patients' also included demographic data and HIV sero status. The study included TB suspects with a negative TB diagnostic history and those who were not on antifungals whilst TB suspects on antifungal therapy that showed improved prognosis and samples of PTB suspects whose request forms lacked demographics were excluded.

\section{Specimen collection / sampling procedure.}

Sputum samples were collected from the Medical ward, TB ward and Outpatient department as instructed by the clinicians under sterile conditions in clean, dry, wide-naked, leak proof containers as per the Uganda national guidelines for tuberculosis infection control in health care facilities, congregate settings 
and households. Sputum samples were then received in the laboratory upon request by the clinicians for PTB test.

The biodata and laboratory results of HIV status of the study participants were recorded corresponding to each participant's sample.

\section{Bacteriological procedure and Mycological Profiling}

Bacterial procedures were predominated by TB diagnosis using Gene xpert which was a diagnostic test for MTB. This was aimed at establishing Mycobacterium aetiology or PTB- fungal co-infection. Fungal examination protocols included a direct wet mount examination with $20 \%$ potassium hydroxide $(\mathrm{KOH})$. Fungal isolation was through cultivation on basic saboruad dextrose agar (SDA) (-/+ antibiotics), incubated at $25^{\circ} \mathrm{C}$ and $37^{\circ} \mathrm{C}$ for 2-4 weeks, and evaluated every 2-3 days. Filamentous fungal isolates unable to vegetatively fruit on SDA were sub-cultured and reisolated on Potato Dextrose Agar (Formedium), incubated and observed as above. Identification was through microscopy by lacto phenol cotton blue staining of fruiting bodies. On the other hand, yeasts were identified biochemically using germ tube method. Definitive diagnosis of pulmonary mycosis was determined based on the presence or absence of fungal elements in a direct examination of culture growth.

\section{Fungal culture}

Sabouraud dextrose agar (SDA) containing antibiotic chloramphenicol and gentamicin was used to culture sputum samples. The specimens were streaked onto the medium in the Universal bottles with a sterile inoculating loop in order to obtain isolated colonies. The preparations were then incubated at $25-$ $30^{\circ} \mathrm{C}$ in an inverted position (agar side up) in humidity conditions. Cultures were examined at least weekly for fungal growth and held for 4 weeks before being reported as negative. After sufficient incubation, the Universal bottles that showed isolated colonies in streaked areas and confluent growth in areas of heavy inoculation were identified and the growth was examined using other methods.

\section{GeneXpert}

The Cepheid GeneXpert @ System was used to detect Mycobacterium tuberculosis for the diagnosis of PTB.

This method was used as a gold standard to diagnosis TB in sputum samples. The sputum samples were first disinfected using Sodium

hypochlorite in a level 2 bio safety cabinet. The prepared samples were then run by the GeneXpert according to the manufacturer's instructions. The GeneXpert MTB/RIF detects DNA sequences specific for Mycobacterium tuberculosis and rifampicin resistance by polymerase chain reaction It is based on 
the Cepheid GeneXpert system, a platform for rapid and simple-to-use nucleic acid amplification tests (NAAT). The Xpert ${ }^{\circledR}$ MTB/RIF purifies and concentrates Mycobacterium tuberculosis bacilli from sputum samples, isolates genomic material from the captured bacteria by sonication and subsequently amplifies the genomic DNA by PCR. The process identifies most of the clinically relevant Rifampicin resistance inducing mutations in the RNA polymerase beta (rpoB) gene in the Mycobacterium tuberculosis genome in a real time format using fluorescent probes called molecular beacons. Results are obtained from unprocessed sputum samples in 90 minutes, with minimal biohazard and very little technical training required operating.

\section{Potassium hydroxide (KOH) mounts}

A drop of $10 \% \mathrm{KOH}$ solution was placed on a slide. A small portion of specimen (sputum) was transferred on to the drop of $\mathrm{KOH}$ and covered with glass. The slide was placed for 15 minutes on a damp cotton wool to prevent the preparation from drying out. Ensured that the material of the preparation was cleared.

The preparation was then examined microscopically using the 10X and 40X objectives with the condenser iris diaphragm closed sufficiently to give a good contrast to detect the fungal elements.

\section{Lacto phenol cotton blue staining.}

LPCB was used for microscopic identification and characterization of fruiting bodies such conidia, sporangia, rhizoids and hypha or mycelia of cultivated fungi on SDA. A drop of lactophenol cotton blue stain was placed on a clean grease-free glass slide. A small fragment of cottony, woolly, or powdery colony was picked from the midpoint of the culture using a sterile straight wire and placed on clean glass slides for the staining process. A clean coverslip was applied avoiding air bubbles. Excess stain was removed with blotting paper and the preparation examined using $\times 10$ and $\times 40$ objectives of the microscope. Fungal element features like microconidia, macroconidia, chlamydospores, and hyphae that appear spiral, pertinate, and antler-like structures were investigated. These features seen in the stained slide were compared with established characteristic fungal features using mycology atlases.

\section{Germ tube test}

Germ-tube test was a simple, reliable procedure for the identification of Candida albicans. $0.5-1 \mathrm{ml}$ of human serum was put into a $12 \times 75 \mathrm{~mm}$ test tube and the yeast colonies were suspended into the serum to obtain faintly turbid suspension. The preparation was then incubated in the tubes at $37^{\circ} \mathrm{C}$ for $2-3$ hours in an incubator. Using sterile Pasteur pipette, the suspension was removed and examined microscopically for the presence or absence of germ tubes. Positive test showed Germ tubes arising directly from the yeast cell and had parallel walls without any constriction at their point of origin which was diagnostic for $C$. albicans. 


\section{Safety and environment}

All biological specimens, including used cartridges, are capable of transmitting infectious agents and thus, were treated with universal precautions. All laboratory procedures were done in a level 2 TB laboratory. Personal protective equipment such as disposable gloves, laboratory coats were used when handling specimens and reagents. Washing of hands were done thoroughly after handling specimens and test reagents. Disposing of used Xpert MTB/RIF cartridges was done according to the country's safety guidelines for hazardous material.

\section{Quality control measures}

Only early morning sputum samples were accepted for analysis so as to easily detect Mycobacterium tuberculosis. Known standard fungal element morphologies and reference cultured fungal elements growth were used for reference. For example, known fungal atlases were used to confirm established characteristic fungal features.

\section{Ethical Consideration.}

The proposal was submitted to the Department of Microbiology, the Faculty of Medicine Review committee (FRC) and approved by the Institutional Review Committee (IRC) of Mbarara University of Science and Technology.

\section{Results}

\section{Demographic distribution, PTB, PFI and PTB-Fungal co-existence profiles}

From a total of 113 sputum samples collected among TB suspects, $4.4 \%(n=5)$ of them produced a positive GeneXpert for MTB, 20.4\% ( $n=23)$ were positive for pulmonary fungal infections (PFI) via direct examination while $70.7 \%(n=80)$ yielded a positive fungal growth via culture which was the diagnostic test for PFI. And, $0.0176 \%(n=2)$ presented with a mixed infection of pulmonary TB -fungal co-existence (Table 1). Based on the findings of this study, the prevalence of TB was more in males $80.4 \%(n=4)$ as opposed to females $(n=1)$. While taking the average of $\mathrm{KOH}$ and culture results, the prevalence of PFI was more in females $62.135 \%(n=31)$, and the only two mixed infections detected were seen only in males (Table 1). The mean age of the participants was $41.91 \pm 15$ years and the minimum and maximum ages were 11 and 84 years respectively. The highest incidence of both PTB and PFI was found in patients aged 18-34 and 35-64 years respectively. Similarly, so, the only mixed infections detected were also in the same age category (Table 1 ).

Table 1: Demographic distribution, Pulmonary tuberculosis (PTB), Pulmonary fungal infections (PFI) and PTB-fungal co-existence profiles 


\begin{tabular}{|c|c|c|c|c|c|}
\hline \multirow[t]{2}{*}{ Variable } & & \multirow{2}{*}{$\begin{array}{l}\text { PTB } \\
\text { profile } \\
\text { GeneXpert } \\
(n=5)\end{array}$} & \multicolumn{2}{|c|}{ Mycological profile } & $\begin{array}{l}\text { PTB-Fungal co- } \\
\text { existence profile }\end{array}$ \\
\hline & & & $\begin{array}{l}\mathrm{KOH}+\mathrm{CFW} \\
(\mathrm{n}=23)\end{array}$ & $\begin{array}{l}\text { Culture } \\
(n=80)\end{array}$ & $\begin{array}{ll}\text { PTB-PFI } & c 0- \\
\text { existance } & n=2\end{array}$ \\
\hline $\begin{array}{l}\text { Age group } \\
\text { (Years) }\end{array}$ & $\begin{array}{l}\text { Proportion } \\
\text { (\%) }\end{array}$ & $\begin{array}{l}\text { Proportion } \\
\text { (\%) }\end{array}$ & $\begin{array}{l}\text { Proportion } \\
\text { (\%) }\end{array}$ & $\begin{array}{l}\text { Proportion } \\
\text { (\%) }\end{array}$ & $\begin{array}{l}\text { Proportion } \\
\text { (\%) }\end{array}$ \\
\hline Children (1-13) & $3(2.7)$ & $0(0.0)$ & $1(4.5)$ & $1(1.25)$ & $0(0.0)$ \\
\hline $\begin{array}{l}\text { Adolescents } \\
(14-17)\end{array}$ & $0(0.0)$ & $0(0.0)$ & $0(0.0)$ & $0(0.0)$ & $0(0.0)$ \\
\hline Youth (18-34) & 38 (33.6) & $2(40.0)$ & $3(13.0)$ & $29(36.25)$ & $1(50.0)$ \\
\hline $\begin{array}{l}\text { Middle aged } \\
(35-64)\end{array}$ & $61(54.0)$ & $2(40.0)$ & $16(69.6)$ & $44(55.0)$ & $1(50.0)$ \\
\hline Elderly (>64) & $11(9.7)$ & $1(20.0)$ & $3(13.0)$ & $6(7.5)$ & $0(0.0)$ \\
\hline \multicolumn{6}{|l|}{ Gender } \\
\hline Male & $46(40.7)$ & $4(80.0)$ & $8(34.8)$ & $33(41.25)$ & $2(100)$ \\
\hline Female & 67 (59.3) & $1(20.0)$ & $15(65.52)$ & $47(58.75)$ & $0(0.0)$ \\
\hline
\end{tabular}

\section{Prevalence of PTB and PFI by HIV status}

HIV/AIDs is a major predisposing factor to both Mycobacterium tuberculosis (MTB) and many of the saprophytic and environmental fungal opportunists. Yet, this can be dependent of the CD4 count of an individual, a parameter that we did not establish here. However, depending on the HIV sero status of our study participants obtained through their medical history, majority of the participants were sero positive $70.8 \%(n=80), 23.09 \% 9(n=26)$ sero negative, while $6.19 \%(n=7)$ of them had an un known sero status for HIV. We further established that both pulmonary tuberculosis $60 \%(n=3)$ and; pulmonary fungal infections $56.5 \%$ by $\mathrm{KOH}$ and $71.3 \%$ by fungal culture were more prevalent in the sero positive cohort as opposed to the sero native (0.00\% PTB; and $21.7 \%$ by $\mathrm{KOH}$ and $22.5 \%$ by fungal culture for PFI) ; and unknown (40.0\% PTB; and $21.7 \%$ by $\mathrm{KOH}$, and $6.25 \%$ by fungal culture for PFI) cohorts; Table 2.

Table 2: Prevalence of pulmonary tuberculosis and pulmonary fungal infections by HIV sero status 


\begin{tabular}{|c|c|c|c|}
\hline Variable & Number (\%) & & \\
\hline \multicolumn{4}{|l|}{ HIV sero-status } \\
\hline Sero-positive & \multicolumn{3}{|l|}{$80(70.8 \%)$} \\
\hline Sero-Negative & \multicolumn{3}{|l|}{$26(23.09 \%)$} \\
\hline Unknown & \multicolumn{3}{|l|}{$7(6.19 \%)$} \\
\hline Total & \multicolumn{3}{|l|}{$113(100 \%)$} \\
\hline \multirow[t]{2}{*}{ Prevalence by HIV sero-status } & PFI & & PTB \\
\hline & $\mathrm{KOH}(\mathrm{n}=23)$ & Culture $(n=80)$ & GeneXpert $(n=5)$ \\
\hline Sero-positive $(n=80)$ & $13(56.5 \%)$ & $57(71.3 \%)$ & $3(60.0 \%)$ \\
\hline Sero-Negative $(n=26)$ & $5(21.7 \%)$ & $18(22.5 \%)$ & $0(0.00 \%)$ \\
\hline Unknown (n=7) & $5(21.7 \%)$ & $5(6.25 \%)$ & $2(40.0 \%)$ \\
\hline HIV and PTB- Fungal co-infection $(n=2)$ & $1(50.0 \%)$ & $1(50.0 \%)$ & $2(100 \%)$ \\
\hline
\end{tabular}

\section{Aetiological profile of pulmonary fungal infections and PTB-PFI co-existence}

Categorically, fungal aetiology was dominated by filamentous fungi $58.75 \%(n=47)$. And, in about $3.75 \%$ $(n=3)$ of the patients a mixed infection of yeasts and filamentous infections was also detected; Figure 1A. According to this study fungal aetiology $70.8 \%(n=80)$ was by far the most prevalent when compared to PTB aetiology $4.4 \%(n=5)$. However, $0.17 \%(n=2)$ of the patients presented with PTB-PFI co-infection; Figure 1B. This was unlikely but interesting finding which needs to be investigated further. On the account of individual aetiology, C. albicans $22.58 \%(n=21)$ was the most prevalent fungi to cause pulmonary fungal infections in this group of patients. This followed closely by Aspergillus spp $17.20 \%(n=16)$, Non albicans candida (NAC) $11.83 \%(n=11)$ and Penicillium spp 10.75\% $(n=10)$. Other, somewhat common aetiological agents also included Trichophyton spp 9.68\% ( $n=9)$, Bipolaris spp $8.60 \%(n=8)$, Acremonium spp 5.38\% $(n=5)$, and Lasiodiplodia spp 3.23\% $(n=3)$. The less popular ones included Sarcinomyces spp, Rhodotorula spp, Mucorspp, Geotrichum candidium, Curvularia lunata , Fusarium spp and Rhizopus spp each of which had a $1.08 \%(n=1)$ representation; Figure $1 C$. As regards PTB-Fungal co- existence, $C$. albicans was the only aetiological agent isolated from the two cases of a possible PTB -fungal coinfection.

\section{Mixed infections, co-infections or co-existence by fungal pathogens}

In the wider environment, fungi co-exist and interacts with similar or other microbes to form different kinds of relationships including the highly addictive mutualistic or endosymbiotic interactions, whilst others can be of antagonistic nature. However, the impact of such interactions in an infection niche on clinical outcomes remains unclear. Through this study, we were able to establish what seemed like a mixed, a co-infection or simply a co-existence by more than one organism in an infection niche in a bout 
$15.9 \%(n=18)$ of the patients. We were able to determine a co-infection of PTB+PFI in $11.11(n=2)$, mixed infection or co-existence of yeasts and filamentous fungi in $16.7 \%(n=3)$, of different yeasts' aetiology in $5.56 \%(n=1)$ and of different filamentous fungal aetiology in $44.4 \%(n=8)$, Table 3 . In regard to age, gender and HIV sero status, the youth (18-34) and middle aged (35-64), females and the sero positive cohorts were the most affected by mixed and co-infections. Individual aetiological agents involved included mainly $C$. albicans predominantly among the PTB+PFI co-infections and yeast-filamentous fungal, and different yeast aetiological mixed infections. On the other hand, Aspergillus spp was predominant among the different filamentous fungal aetiological mixed infections, Figure 2:

Table 3: Aetiology of mixed and co-infections by age, gender and sero status 
Variable Mixed infections, co-infections or co-existence by fungal pathogens

$(n=18)$

PTB + PFI Yeast + Filamentous Different Yeasts Different filamentous

$(n=2)$

fungal aetiology

aetiology

fungi aetiology

$(11.11 \%) \quad(n=3)(16.7 \%)$

$(n=1)(5.56 \%)$

$(n=8)(44.4 \%)$

Proportion Proportion (\%)

(\%)

Proportion (\%) Proportion (\%)

\section{Age group}

\begin{tabular}{|c|c|}
\hline Children (1- & $0(0.00)$ \\
\hline
\end{tabular}

13)

$\begin{array}{lcccc}\begin{array}{l}\text { Adolescents } \\ \text { (14-17) }\end{array} & 0(0.00) & 1(5.56) & 0(0.00) & 0(0.00) \\ \begin{array}{l}\text { Youth (18- } \\ \text { 34) }\end{array} & 1(5.56) & 1(5.56) & 0(0.00) & 5(27.8) \\ \begin{array}{l}\text { Middle aged } \\ \text { (35-64) }\end{array} & 1(5.56) & 1(5.56) & 1(5.56) & 3(16.7)\end{array}$

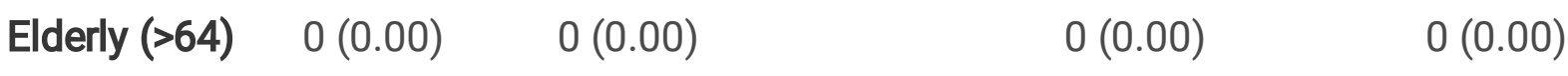

\section{Gender}

$\begin{array}{lllll}\text { Male } & 2(11.1) & 0(0.00) & 0(0.00) & 2(11.1) \\ \text { Female } & 0(0.00) & 3(16.7) & 1(5.56) & 6(33.3)\end{array}$

\section{HIV sero}

status

\begin{tabular}{lcccc}
$\begin{array}{l}\text { Sero } \\
\text { positive }\end{array}$ & $1(5.56)$ & $3(16.7)$ & $1(5.56)$ & $7(38.9)$ \\
$\begin{array}{l}\text { Sero } \\
\text { negative }\end{array}$ & $0(0.00)$ & $0(0.00)$ & $0(0.00)$ & $1(5.56)$ \\
\hline Un known & $1(5.56)$ & $0(0.00)$ & $0(0.00)$ & $0(0.00)$
\end{tabular}

\section{Discussion}

For some time now pulmonary TB has been established as a major opportunistic pulmonary disease especially in the HIV/AIDs infected cohort with about 2 million people dying and 1 million infected worldwide each year (12). However, in as many parts of the world endemic pulmonary mycoses particularly the deep seated ones are also prevalent and just like TB, are also responsible for high rates 
of morbidity and mortality in an array of patient populations' (13) (14-16). The challenge has always been that in addition to the fact that both entities present with similar symptoms, patients with a history of suffering from TB have proven prone to certain opportunistic fungal infections such as Aspergillus. For instance, chronic aspergillosis commonly complicates treated pulmonary tuberculosis with residual cavitation (17). However, in many parts of the world the medical importance of pulmonary fungal infections continues to be ignored in the at-risk individuals (18).

This brief background highlights the medical importance of both PTB and pulmonary mycoses. Yet the role of opportunistic fungi as secondary invaders of lungs, kidneys and other organs of patients having underlying conditions such as HIV/AIDS and cancer is somewhat documented to a considerable variation tune of $9-80 \%$ incidence reported in literature (18). In Uganda though, there is a general paucity of the burden of pulmonary mycoses. As a result, these infections have remained a silent challenge to public health due to the fact that they have either been neglected, ignored, missed or misdiagnosed $(10,19,20)$. The spectrum of pulmonary fungal aetiology has been evolving over the years from the commonly isolated candida species and primary dimorphic fungi to now several saprophytic molds and dematiaceous fungi. Although C. albicans is still the most common, infections due to other members of the genus and other fungal categories are now on the increase. However, fungal diagnosis still remains a challenge in most health centers with limited diagnostic tools and very few trained mycologists to counter this challenge.

In this study, the fungal aetiology prevalence using culture on SDA as our diagnostic test was 80 (70.8\%) this dominated $5(4.4 \%)$ prevalence of MTB aetiology. The low TB prevalence here is interesting to know and it would have been important to establish if some of these patients that presented with a fungal infection had a history of TB treatment. The prevalence of pulmonary mycoses according to gender was higher in females at $62.135 \%(n=31)$. The highest incidence of both PTB and PFI was found in patients aged 18-34 and 35-64 years respectively. Similarly, so, the only mixed infections detected were also in the same age category (Table1). These findings are in accordance with study by (21) and higher than study done by (22). This difference in prevalence can be attributed to geographical location, sample size and diagnostic approaches used. On the other hand the prevalence of TB was $5(4.4 \%)$ (Table 1 ) higher than findings of study by $(23)$ but lower than findings by $(24,25)$.

In this study, $C$. albicans was the predominant isolate with a prevalence of $22.58 \%$ followed by Aspergillus $17.20 \%$, Non C. albicans $11.83 \%$, Penicillium species $10.73 \%$, Trichophyton species $9.68 \%$, Bipolaris species $8.60 \%$, Acremonium species $5.38 \%$, Lasiodiplodia species $3.23 \%$ (Figure 2). These findings are similar to several studies including (26) that have named $C$. albicans and Aspergillus among the dominating aetiological agents of pulmonary mycosis. However, the aetiological profile determined is interestingly different than most studies (22). For instance, the next most common aetiological agents other than yeasts and Aspergillus species were Penicillium species, Trichophyton species and Bipolaris species. These are dematiaceous ascomycotina fungi that are often associated with soil and plant debris. Other rare opportunistic pathogens in addition to other isolates included Sarcinomyces Mucor, Fusarium, Rhodotorula, Curvularia lunata, Rhizopus, Geotrichum candidum, each accounting for $1.08 \%$

Page $12 / 18$ 
and the dermatophytes are isolates in this infection niche. However, their isolation here could be linked to patients underlying conditions, exposure, geographical location and perhaps evolution in microbial adaptions mechanisms. Indeed, the aetiological profile established in this study should send out a strong message regarding fungal aetiological evolution over the past few decades.

On the other hand it's not uncommon for fungi to co-exist with other microbes in the same infection niche. In this study, we also sought to establish the prevalence of PTB, PFI and fungal-PTB co-infections among the PTB suspects by HIV sero status. We established a prevalence of 57 (71.3\%) for PFI, $3(60.0 \%)$ for MTB in HIV sero positive patients and 18 (22.5\%) for PFI in HIV sero negative patients and $0(0.00 \%)$ for MTB in HIV sero negative patients. On the other hand, 2 (100\%) sero positive patients were co-infected with both PFI and MTB (Table 2). These results prove the fact that HIV/AIDs is a major predisposing factor to both Mycobacterium tuberculosis (MTB) and many of the saprophytic and environmental fungal opportunists and also there is existing evidence that individuals treated for PTB are prone to fungal infection (17).

In the wider environment, fungi co-exist and interact with similar or other microbes to form different kinds of relationships including the highly addictive mutualistic or endosymbiotic interactions, whilst others can be of antagonistic nature. However, the impact of such interactions in an infection niche on clinical outcomes remains unclear. Through this study, we were able to establish what seemed like a mixed, a coinfection or simply a co-existence by more than one organism in an infection niche in about $15.9 \%(n=18)$ of the patients. We were able to determine a co-infection of PTB+PFI in $11.11 \%(n=2)$, mixed infection or co-existence of yeasts and filamentous fungi in $16.7 \%(n=3)$, of different yeasts' aetiology in $5.56 \%(n=1)$ and of different filamentous fungal aetiology in $44.4 \%(n=8)$, Table 3 . In regard to age, gender and HIV sero status, the youth (18-34) and middle aged (35-64), females and the sero positive cohorts were the most affected by mixed and co-infections. Individual aetiological agents involved mainly $C$. albicans predominantly among the PTB+PFI co-infections and yeast-filamentous fungal and different yeast aetiological mixed infections. On the other hand, Aspergillus spp was predominant among the different filamentous fungal aetiological mixed infections, Figure 2: PFI and PTB co-infection prevalence of $11.11 \%$ in this study was apparently higher than the one found in a study done by (27). In addition, we established $C$. albicans as the common fungus associated with TB-Fungal co-infection similar to findings by (28-31) but different from studies by $(32,33)$, however in our study no Aspergillus species were found to co-infect with PTB.

As already shown by prior studies, our findings here continue to highlight the medical importance of pulmonary fungal infections among patients suspected for TB. Most importantly, the aetiological diversity established here is rich enough to suggest precise examination using different methods. What is important to note here though is that the established aetiological scope is predominated by opportunistic fungus which in essence are not as grossly damaging by themselves however when superimposed on to certain underlying conditions such as TB, their effects can be fatal. Thus, the need to be aware of this possibility in order to up appropriate strategies for prevention, diagnosis, and management in TB suspected individuals is now apparent. 


\section{Declarations}

\section{Availability of data and materials}

Data and materials are readily available from the corresponding author upon request.

\section{Authors' contributions}

IKN, MP, and AM contributed in study conception and design, KK and JM Collected data and participated in laboratory analysis, JT and EN carried data cleaning, LA and BM carried out data analysis, IKN, BA and $\mathrm{HI}$ wrote the first draft of the manuscript while JK, TK reviewed the manuscript and JB supervised the whole research process.

\section{Conflicts of Interest}

The authors declare that they have no conflicts of interest

\section{Acknowledgments}

We are grateful to the study participants and staff Mbarara University Microbiology department for all the support given to us during data collection

\section{Funding}

We did not receive any grant to fund this study.

\section{References}

1. Kyu HH, Maddison ER, Henry NJ, Mumford JE, Barber R, Shields C, et al. The global burden of tuberculosis: results from the Global Burden of Disease Study 2015. The Lancet Infectious Diseases. 2018;18(3):261-84.

2. Буйневич И, Гопоняко С. Tuberculosis= Туберкулез. 2019.

3. MacNeil A, Glaziou P, Sismanidis C, Maloney S, Floyd K. Global epidemiology of tuberculosis and progress toward achieving global targets-2017. Morbidity and Mortality Weekly Report. 2019;68(11):263.

4. WHO. Global Tuberculosis Report. 2020. 2020.

5. Ministry of Health U. National Tuberculosis and Leprosy Division July 2017 - June 2018 Report. 2018:10.

6. Holt MR, Chan ED. Chronic cavitary infections other than tuberculosis. Journal of thoracic imaging. 2018;33(5):322-33.

7. Warnock DW. Trends in the epidemiology of invasive fungal infections. Nippon Ishinkin Gakkai Zasshi. 2007;48(1):1-12. 
8. Chrdle A, Mallátová Na, Vašáková M, Haber J, Denning DW. Burden of serious fungal infections in the Czech Republic. Mycoses. 2015;58:6-14.

9. US K. Denning DW. Burden of serious fungal infections in Nepal. Mycoses. 2015;58:45-50.

10. Bongomin F, Gago S, Oladele RO, Denning DW. Global and multi-national prevalence of fungal diseases-estimate precision. Journal of fungi. 2017;3(4):57.

11. Kalyani CS, Koripella RL, Madhu C. Fungal isolates in sputum samples of multidrug-resistant tuberculosis suspects. Int J Sci Stud. 2016;4(2):164-6.

12. De Backer Al, Mortelé KJ, De Keulenaer BL, Parizel PM. Tuberculosis: epidemiology, manifestations, and the value of medical imaging in diagnosis. JBR-BTR : organe de la Societe royale belge de radiologie $(\mathrm{SRBR})=$ orgaan van de Koninklijke Belgische Vereniging voor Radiologie (KBVR). 2006;89(5):243-50.

13. Di Mango AL, Zanetti G, Penha D, Menna Barreto M, Marchiori E. Endemic pulmonary fungal diseases in immunocompetent patients: an emphasis on thoracic imaging. Expert review of respiratory medicine. 2019;13(3):263-77.

14. Wheat LJ, Goldman M, Sarosi G, editors. State-of-the-art review of pulmonary fungal infections. Seminars in respiratory infections; 2002.

15. Lass-Flörl C. The changing face of epidemiology of invasive fungal disease in Europe. Mycoses. 2009;52(3):197-205.

16. Brown GD, Denning DW, Gow NA, Levitz SM, Netea MG, White TC. Hidden killers: human fungal infections. Science translational medicine. 2012;4(165):165rv13-rv13.

17. Page ID, Byanyima R, Hosmane S, Onyachi N, Opira C, Richardson M, et al. Chronic pulmonary aspergillosis commonly complicates treated pulmonary tuberculosis with residual cavitation. The European respiratory journal. 2019;53(3).

18. Astekar M, Bhatiya PS, Sowmya GV. Prevalence and characterization of opportunistic candidal infections among patients with pulmonary tuberculosis. Journal of oral and maxillofacial pathology : JOMFP. 2016;20(2):183-9.

19. Parkes-Ratanshi R, Achan B, Kwizera R, Kambugu A, Meya D, Denning D. Cryptococcal disease and the burden of other fungal diseases in Uganda; Where are the knowledge gaps and how can we fill them? Mycoses. 2015;58:85-93.

20. Tufa TB, Denning DW. The burden of fungal infections in Ethiopia. Journal of Fungi. 2019;5(4):109.

21. Aluyi $\mathrm{H}$, Otajevwo $\mathrm{F}$, Iweriebor $\mathrm{O}$. Incidence of pulmonary mycoses in patients with acquired immunodeficiency diseases. Nigerian Journal of Clinical Practice. 2010;13(1).

22. Ekenna O, Uba A, Chikwem J, Mambula S, Aliyu M, Mohammed I. Relevance of moldy fungi as agents of chronic lower respiratory tract infection in patients seen in Maiduguri, Nigeria. West African journal of medicine. 2007;26(2):117-20.

23. Buthia T, Adhikari L. Pulmonary mycoses among the clinically suspected cases of pulmonary tuberculosis. International Journal of Research in Medical Sciences. 2015;3(1):260-8. 
24. Njunda AL, Ewang AA, Kamga L-HF, Nsagha DS, Assob J-CN, Ndah DA, et al. Respiratory tract Aspergillosis in the sputum of patients suspected of tuberculosis in Fako division-Cameroon. $J$ Microbiol Res. 2012;2(4):68-72.

25. Chadeganipour M, Shadzi S, Dehghan P, Bijary J. The incidence of opportunistic fungi in patients suspected of tuberculosis. Mycoses. 2000;43(7-8):269-72.

26. Nawange $M$, Kavishwar A. Prevalence of opportunistic fungal infection in patients with pulmonary tuberculosis in Madhya Pradesh, Central India. J Microbiol Biomed Res. 2015;1:6.

27. Yahaya H, Taura D, Aliyu I, Bala J, Yunusa I, Ahmad I, et al. Spectrum of opportunistic mould infections in suspected pulmonary tuberculosis (TB) patients. International Journal of Microbiology and Application. 2015;2(1):6-11.

28. Astekar M, Bhatiya PS, Sowmya G. Prevalence and characterization of opportunistic candidal infections among patients with pulmonary tuberculosis. Journal of oral and maxillofacial pathology: JOMFP. 2016;20(2):183.

29. Mathavi S, Shankar R, Kavitha A, Sasikala G, Priyadharsini I. A study on prevalence of pulmonary candidiasis among tuberculosis patients and use of chromagar in identification of Candida species. Journal of Drug Delivery and Therapeutics. 2014;4(3):118-21.

30. Hadadi-Fishani M, Shakerimoghaddam A, Khaledi A. Candida coinfection among patients with pulmonary tuberculosis in Asia and Africa; A systematic review and meta-analysis of cross-sectional studies. Microbial Pathogenesis. 2020;139:103898.

31. Amiri MRJ, Siami R, Khaledi A. Tuberculosis status and coinfection of pulmonary fungal infections in patients referred to reference laboratory of Health Centers Ghaemshahr City during 2007-2017. Ethiopian journal of health sciences. 2018;28(6).

32. Osman NM, Gomaa AA, Sayed NM. Microarray detection of fungal infection in pulmonary tuberculosis. Egyptian Journal of Chest Diseases and Tuberculosis. 2013;62(1):151-7.

33. Bansod S, Rai M. Emerging of mycotic infection in patients infected with Mycobacterium tuberculosis. World J Med Sci. 2008;3(2):74-8.

\section{Figures}




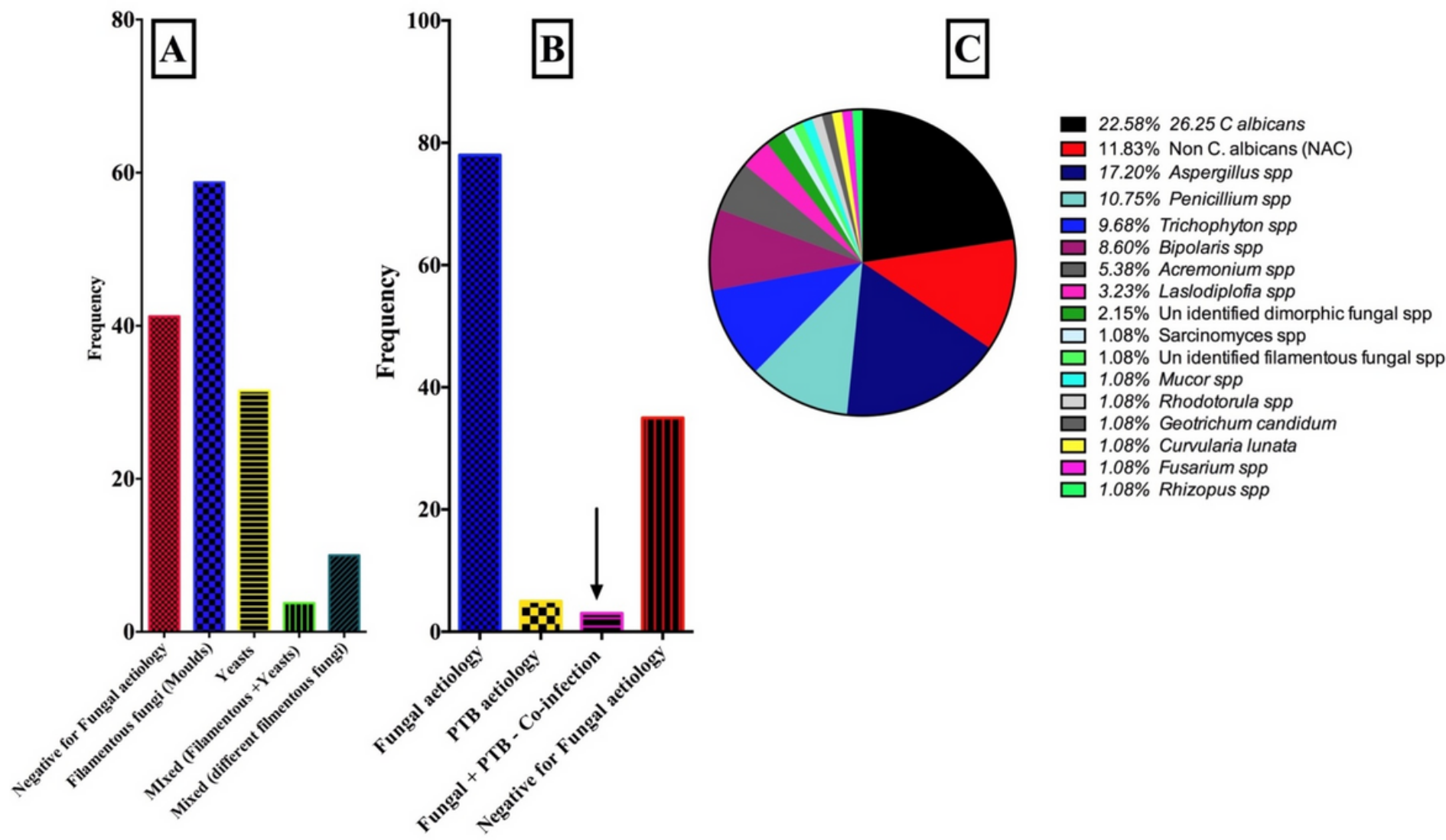

Figure 1

Aetiological profile of pulmonary fungal infections and PTB -PFI co-infections 


\section{Mixed and co-infections aetiology}

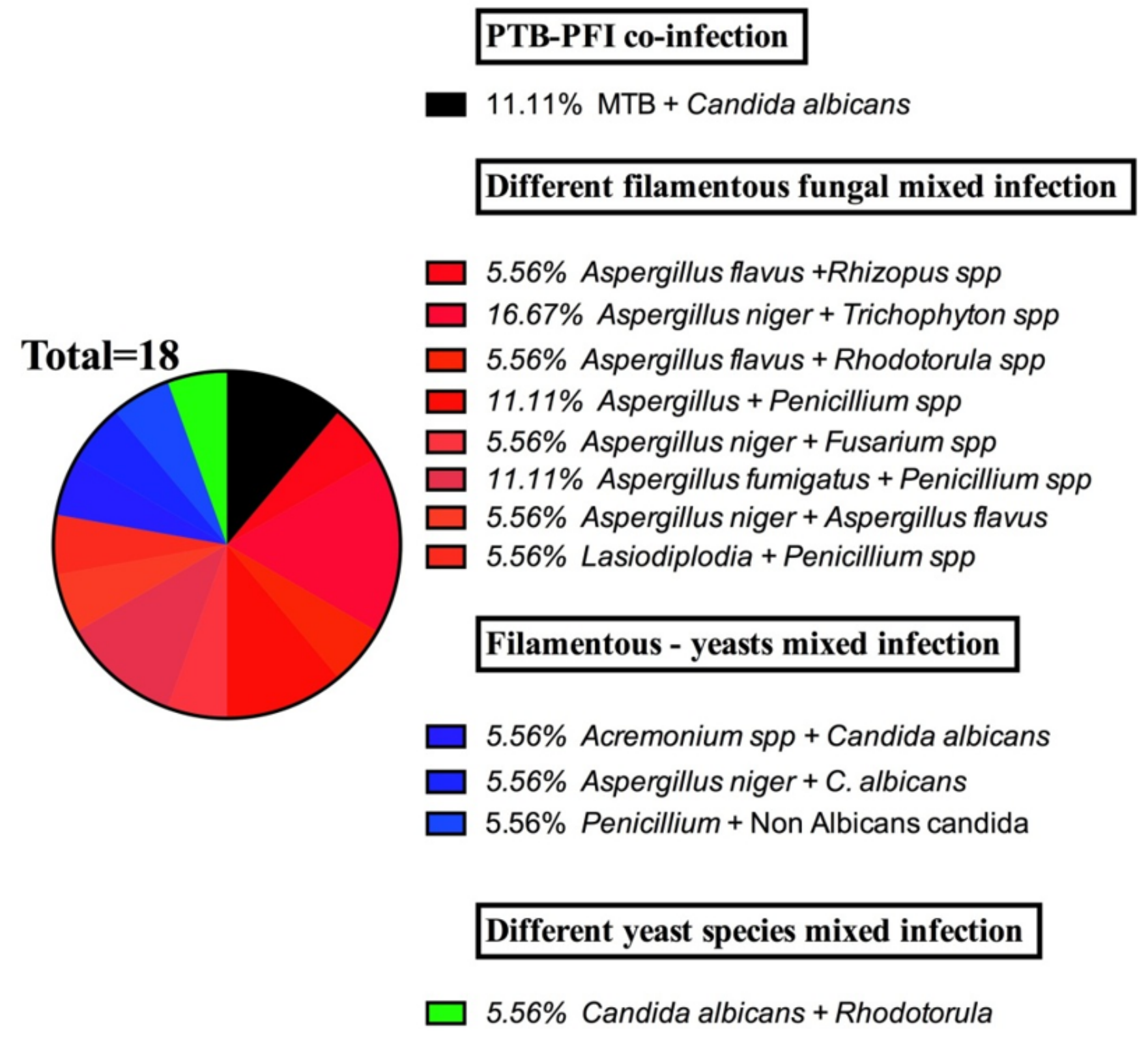

Figure 2

Aetiology of mixed and co-infections 